\title{
Long-acting inhalable chitosan-coated poly(lactic- co-glycolic acid) nanoparticles containing hydrophobically modified exendin-4 for treating type 2 diabetes
}

This article was published in the following Dove Press journal: International Journal of Nanomedicine

8 August 2013

Number of times this article has been viewed

\author{
Changkyu Lee' \\ Ji Su Choil \\ Insoo Kim' \\ Kyung Taek $\mathrm{Oh}^{2}$ \\ Eun Seong Lee ${ }^{3}$ \\ Eun-Seok Park' \\ Kang Choon Lee' \\ Yu Seok Youn' \\ 'School of Pharmacy, Sungkyunkwan \\ University, Suwon, Republic of Korea; \\ ${ }^{2}$ College of Pharmacy, Chung-Ang \\ University, Seoul, Republic of Korea; \\ ${ }^{3}$ Division of Biotechnology, The \\ Catholic University of Korea, \\ Bucheon-si, Republic of Korea
}

\begin{abstract}
Inhalable glycol chitosan-coated poly(lactic-co-glycolic acid) (PLGA) nanoparticles containing palmitic acid-modified exendin-4 (Pal-Ex4) (chitosan Pal-Ex4 PLGA NPs) were prepared and characterized. The surface morphology, particle size, and zeta potential of chitosan Pal-Ex4 PLGA NPs were investigated, and the adsorption and cytotoxicity of chitosan Pal-Ex4 PLGA NPs were evaluated in human lung epithelial cells (A549). Finally, the lung deposition characteristics and hypoglycemia caused by chitosan Pal-Ex4 PLGA NPs were evaluated after pulmonary administration in imprinting control region (ICR) and type 2 diabetic $\mathrm{db} / \mathrm{db}$ mice. Results showed that chitosan Pal-Ex4 PLGA NPs were spherical, compact and had a diameter of $\sim 700 \mathrm{~nm}$ and a positive surface charge of $+28.5 \mathrm{mV}$. Chitosan-coated PLGA NPs were adsorbed onto A549 cells much more so than non-coated PLGA NPs. Pal-Ex4 release from chitosan-coated PLGA NPs was delayed by as much as 1.5 days as compared with chitosan-coated Ex4 PLGA NPs. In addition, chitosan-coated PLGA NPs remained in the lungs for $\sim 72$ hours after pulmonary administration, whereas most non-coated PLGA NPs were lost at 8 hours after administration. Furthermore, the hypoglycemic efficacy of inhaled chitosan Pal-Ex4 PLGA NPs was 3.1-fold greater than that of chitosan Ex4 PLGA NPs in $\mathrm{db} / \mathrm{db}$ mice. The authors believe chitosan Pal-Ex4 PLGA NPs have considerable potential as a long-acting inhalation delivery system for the treatment of type 2 diabetes.
\end{abstract}

Keywords: chitosan-coating, PLGA nanoparticles, inhalation, exendin-4, type 2 diabetes

\section{Introduction}

The systemic delivery of peptide or protein drugs via the pulmonary route has many advantages over other non-parenteral routes, such as oral, buccal, nasal, or rectal routes, because the lungs offer favorable physiological features for drug absorption, ${ }^{1}$ such as a large surface area $\left(\sim 75 \mathrm{~m}^{2}\right)$, a thin epithelial barrier $(0.1-0.5 \mu \mathrm{m}$ in alveoli), slow mucociliary clearance, and insignificant enzymatic activity. ${ }^{2,3}$ Therefore, the inhalation route has attracted great interest as a noninvasive systemic means of access for peptide and protein treatments used to treat diabetes. ${ }^{4-8}$ Furthermore, patient compliance is low because most anti-diabetic drugs are still administered by injection and diabetes requires long-term therapy.

Nanoparticles have many obvious merits as a pulmonary delivery system, as they provide sustained drug release and are able to move to the alveolar epithelium, whereas larger, more compact microparticles are not. ${ }^{9}$ Moreover, nanoparticles of $<0.26 \mu \mathrm{m}$ are not severely phagocytosed in alveoli or rapidly eliminated by mucociliary clearance. ${ }^{10,11}$
School of Pharmacy, Sungkyunkwan University, 300 Cheoncheon-dong, Jangan-gu, Suwon 440-746,

Republic of Korea

Tel +82 3I 2907785

Fax +82312907724

Email ysyoun@skku.edu 
In fact, inhalable nanoparticles composed of glycol chitosan, poly(lactic acid-co-glycolic acid), or polybutylcyanoacrylate have been shown to have sustained-release characteristics for insulin, calcitonin, and exendin-4 (Ex4) and prolonged therapeutic effects in osteoporosis and diabetes. ${ }^{12-14}$

Chitosan, the $\mathrm{N}$-acetylated derivative of chitin, has been widely used as a building material for nanoparticles. It has excellent biocompatibility and biodegradability characteristics, and little toxicity, presumably because of its natural origin. ${ }^{15-17}$ Furthermore, chitosan prolongs residence times in tissues and aids the absorptions of macromolecules, such as peptides and proteins, because chitosan adheres to mucus and enhances the permeation efficiency of those drugs. ${ }^{9}$ In addition, several authors have demonstrated that nanoparticles composed of chitosan or coated with chitosan substantially increase bioavailability and efficacy when administered via the pulmonary route. , $^{9,13}$

However, despite the many advantages of the pulmonary route, frequent and long-term inhalation is necessary to achieve satisfactory efficacy because the therapeutic effects of most protein drugs are short. Accordingly, a delivery system that allows the long-term release of drugs after a single inhalation is required. To achieve this, we designed sustained-release systems using a derivatized peptide. For example, we found palmitic-acid modified Ex4 (Pal-Ex4) has a prolonged anti-diabetic effect in terms of prolonging hypoglycemia. ${ }^{4,5}$ In the present study, we sought to fabricate inhalable poly(lactic-co-glycolic acid) (PLGA) nanoparticles containing Pal-Ex4. In addition, these nanoparticles were surface-coated with chitosan to increase residence times in lungs based on consideration of its ability to adhere to mucus. Furthermore, the in vivo lung deposition characteristics (via pulmonary intubation) and the hypoglycemic efficacy of the prepared chitosan-coated PLGA nanoparticles containing Pal-Ex4 (chitosan Pal-Ex4 PLGA NPs) were evaluated in a $\mathrm{db} / \mathrm{db}$ diabetic mouse model.

\section{Materials and methods Materials}

Poly(d,1-lactic-co-glycolic acid) (PLGA) (Mw: 10,000; lactic acid : glycolic acid = 50:50) was purchased from Wako Pure Chemical (Tokyo, Japan). Ex4 and N-hydroxysuccinimidylactivated palmitic acid (Pal-NHS) were purchased from the American Peptide Company (Sunnyvale, CA, USA) and Sigma-Aldrich (St Louis, MO, USA), respectively. Chitosan (low Mw: 20,000 cps) was purchased from Sigma-Aldrich. Fluorescein-NHS and Cy5.5 NHS ester were purchased from Pierce (Rockford, IL, USA) and GE Healthcare (Piscataway,
NJ, USA), respectively. All other reagents, unless otherwise specified, were obtained from Sigma-Aldrich.

\section{Animals}

Type 2 diabetic C57BL/6 db/db mice (males, 5-6 weeks old) were purchased from the Korean Research Institute of Bioscience and Biotechnology (Daejon, South Korea). Imprinting control region (ICR) mice (males, $\sim 6$ weeks old) were purchased from the OrientBio Experimental Animal Laboratory (Busan, South Korea). Animals were cared for in accordance with the guidelines issued by the National Institute of Health for the care and use of laboratory animals (NIH publication 80-23, revised in 1996). Animals were housed in groups of 6-8 under a 12-hour light/dark cycle (lights on at $6 \mathrm{am}$ ), allowed food and water ad libitum, and acclimatized for 2 weeks. This study was approved by the Ethical Committee on Animal Experimentation at Sungkyunkwan University.

\section{Preparation of Pal-Ex4}

Pal-Ex4 was prepared using a slight modification of a previously described procedure. ${ }^{6,7,18,19}$ Briefly, $7 \mathrm{mg}$ of Ex4 was mixed with $0.9 \mathrm{mg}$ of Pal-NHS (molar ratio $1: 1.5$ ) in $3 \mathrm{~mL}$ of $0.3 \%$ triethylamine in anhydrous dimethylsulfoxide and allowed to react at room temperature for 90 minutes. The reaction mixture was then subjected to reverse-phase highperformance liquid chromatography (RP-HPLC) on a LiChrospher 100 RP-18 column $(250 \times 4.0$ mm, $5 \mu \mathrm{m}$; Merck KGaA, Darmstadt, Germany) at ambient temperature. Gradient elution was conducted at a flow-rate of $1.0 \mathrm{~mL} /$ minute using solvent A (0.1\% trifluoroacetic acid [TFA] in DW) and solvent B $(0.1 \%$ TFA in acetonitrile) using 30\%-50\% B for 10 minutes and $50 \%-90 \% \mathrm{~B}$ for 20 minutes. Eluates were monitored at $215 \mathrm{~nm}$, and the fraction corresponding to PalEx4 was collected, dried under nitrogen, and stored in $10 \mathrm{mM}$ phosphate buffer saline (PBS, $\mathrm{pH} 7.4)$ at $4^{\circ} \mathrm{C}$ until required.

\section{Preparation of chitosan Pal-Ex4 PLGA NPs}

PLGA nanoparticles containing Ex4 derivatives were prepared using a slight modification of a previously described method. ${ }^{13,20,21}$ Briefly, an aliquot $(200 \mu \mathrm{L})$ of either Ex4 or Pal-Ex4 $(400 \mu \mathrm{g})$ was poured into $1 \mathrm{~mL}$ of ethyl acetate containing PLGA (40 mg). The resulting mixture was emulsified at 10,000 rpm for 4 minutes using a WiseTis HG15D homogenizer (DAIHAN Scientific Co, Seoul, South Korea). Four milliliters of PVA $(5 \%, \mathrm{w} / \mathrm{w})$ was then poured into the emulsion and stirred at 7,000 rpm for 5 minutes, and $30 \mathrm{~mL}$ of deionized water was then added to the resulting $\mathrm{w} / \mathrm{o} / \mathrm{w}$ 
emulsion and stirred at 4,000 rpm for 5 minutes using a Silverson Laboratory Mixer (model L4RT) with a 5/8-inch head (Silverson Machines, Inc, East Longmeadow, MA, USA). The organic phase was allowed to evaporate by gentle stirring overnight, and the Pal-Ex4 or Ex4 PLGA NPs were harvested by centrifugation at $8,000 \mathrm{rpm}$ for 15 minutes. To coat these nanoparticles with chitosan, the whole amount of PLGA NPs harvested were suspended in $50 \mathrm{mM}$ of 2-(N-morpholino) ethanesulfonic acid (MES) buffer ( $\mathrm{pH} 5.5$ ) containing a $6 \mathrm{mg}$ aliquot of chitosan dissolved in $1 \mathrm{~mL}$ of $1 \%(\mathrm{v} / \mathrm{v})$ acetic acid and stirred for 12 hours. Chitosan-coated PLGA NPs were harvested by centrifugation at 8,000 rpm for 15 minutes and washed three times with DW. Chitosan-coated PLGA nanosphere pellets, which were previously dispersed in $10 \mathrm{~mL} \mathrm{DW}$ containing $20 \mathrm{mg}$ trehalose, were further lyophilized.

\section{Characterization of the different PLGA NPs}

The particle sizes and zeta potentials of the prepared chitosancoated or non-coated PLGA NPs containing Ex4 or Pal-Ex4 were measured using a Zetasizer Nano-S90 (Malvern Instruments, Malvern, UK) and a $633 \mathrm{~nm}$ He-Ne laser beam at a fixed scattering angle of $90^{\circ}$. Experiments were performed at a nanosphere concentration of $2 \mathrm{mg} / \mathrm{mL}$ on a PLGA weight basis, and to measure zeta potentials, the nanoparticles were dispersed in DW. The surface morphologies of PLGA NPs containing Ex4 or Pal-Ex4 were observed under a transmission electron microscope (TEM; Hitachi H-7600; Hitachi Ltd, Tokyo, Japan).

\section{Evaluations of the release profiles of Ex4 and Pal-Ex4 from PLGA NPs}

Aliquots ( $1 \mathrm{~mL}, 7 \mathrm{mg}$ ) of chitosan-coated or naïve PLGA NPs containing Ex4 or Pal-Ex4 in $10 \mathrm{mM}$ PBS (pH 7.4) were gently agitated at $37^{\circ} \mathrm{C}$ for $0.5,1.0,2.0$, or 3.0 days and centrifuged at $12,000 \mathrm{rpm}$ for 30 minutes. Supernatants were removed, and the PLGA NPs were extracted using $0.3 \mathrm{~mL}$ of a $1: 1$ mixture solution of $0.1 \%$ TFA acetonitrile and $0.1 \%$ TFA DW by mild agitation for 1 hour. The supernatants obtained by centrifugation at $8,000 \mathrm{rpm}$ for 15 minutes were subjected to HPLC using the procedure mentioned above.

\section{Characterization of the adhesion of PLGA NPs onto A549 cells}

PLGA NPs containing Ex4 derivatives were prepared using a slight modification of a previously described method. ${ }^{10,22,23}$ A549 cells were plated onto 6-well plates at a density of $5.0 \times 10^{6}$ cells/well and incubated for 24 hours to ensure cell adhesion. Briefly, cells were incubated in Roswell Park Memorial Institute (RPMI) 1640 medium supplemented with 10\% (v/v) fetal bovine serum (FBS; Gibco; Life Technologies, Carlsbad, CA, USA) containing 1\% penicillin/streptomycin (Gibco), and maintained in a $5 \% \mathrm{CO}_{2} / 95 \%$ humidified incubator at $37^{\circ} \mathrm{C}$. After seeding, the medium was replaced with RPMI 1640 medium supplemented with 1\% (v/v) FBS containing coumarin 6-loaded PLGA NPs $(0.025 \mathrm{mg} / \mathrm{mL})$. The medium was then removed, and cells were air-dried and fixed with paraformaldehyde (4\% in $10 \mathrm{mM}$ PBS, $\mathrm{pH} 7.4$ ) for 1 hour at room temperature. Slides were then rinsed with PBS. Cells were permeabilized by incubating them with $0.1 \%$ Triton X-100 and $0.1 \% \mathrm{Na}$-citrate for 2 minutes at room temperature, rinsed twice with PBS, dried, and observed under a confocal laser scanning microscope (CLSM; Carl Zeiss Meta LSM510; Carl Zeiss Meditec AG, Jena, Germany).

\section{Aerosolization of chitosan PLGA NPs}

A portion $(50 \mu \mathrm{L})$ of chitosan Pal-Ex4 PLGA NPs was aerosolized using a microsprayer aerosolizer (Model IA-1C-M; Penn-Century, Inc, Philadelphia, PA, USA). Images of the aerosolization were captured at $0.04-0.08$ second intervals after actuation using a digital video camera.

\section{Pulmonary administration of PLGA NPs to mice}

PLGA NPs containing either Ex4 or Pal-Ex4 (Ex4 PLGA NPs or Pal-Ex4 PLGA NPs, respectively) were administered to mice using a modification of a previously described procedure. ${ }^{4,5}$ Briefly, male $\mathrm{db} / \mathrm{db}$ or ICR mice were anesthetized with a single intraperitoneal (ip) injection of Zoletil $(0.5 \mathrm{mg} / \mathrm{kg})$, and $50 \mu \mathrm{L}$ of Ex 4 or Pal-Ex4 PLGA NPs was directly insufflated into lungs via an opening in the trachea using the microsprayer aerosolizer described above. Visualization of the tracheal opening was achieved using an otoscope (Heine Mini 3000; HEINE Optotechnik, Herrsching, Germany).

\section{Evaluation of chitosan-coated PLGA NPs deposition in mouse lungs}

Coumarin 6-incorporated chitosan PLGA NPs (target loading $1 \%$ ) were prepared using the procedure described in above. A $50 \mu \mathrm{L}$ solution of PLGA NPs, either chitosan-coated or noncoated PLGA, containing coumarin $6(5 \mathrm{mg} / \mathrm{mL})$ were directly insufflated into the lungs of ICR mice. At predetermined times $(0,8,24$, and 72 hours), animals were sacrificed, and lung lobes were harvested and monitored using an in vivo imaging system (Optix MX3, ART Advanced Research Technologies Inc, Montreal, Canada) using an emission wavelength of $470 \mathrm{~nm}$. 


\section{Hypoglycemic efficacies of pulmonary administered chitosan Ex4 or Pal-Ex4 PLGA NPs in non-fasted $\mathrm{db} / \mathrm{db}$ mice}

To evaluate hypoglycemic efficacies, chitosan-coated Pal-Ex4 or Ex4 PLGA NPs (100 nmol/kg as Ex4) were administered by insufflation to male $\mathrm{db} / \mathrm{db}$ mice $(\mathrm{n}=6 /$ group, 9 weeks old) previously anesthetized with Zoletil (0.5 mg/kg, ip). Chitosan-coated PLGA NPs were administered as a control. Mice were kept under non-fasting conditions with free access to water and food until the end of experiment, as previously described. ${ }^{6,7}$ A drop of blood was drawn from the tail vein of each mouse at $0,0.5,1,2,4,6,8,12,24,36,48,72$, and 96 hours after administration, and blood glucose levels were determined using a one-touch blood glucose meter (ACCU$\mathrm{CHEK}^{\circledR}$ Sensor, Roche Diagnostics Corp, Indianapolis, IN, USA). Minimum glucose levels and times to reach these levels were noted. In addition, hypoglycemic efficacies were evaluated by calculating total hypoglycemic degrees (defined as area under the curve $\left.[\mathrm{AUC}]_{\text {saline, } 0-96 \text { hours }}-[\mathrm{AUC}]_{\text {test, } 0-96 \text { hours }}\right)$.

\section{Cytotoxicity of PLGA NPs in A549}

The cytotoxicities of chitosan PLGA NPs were evaluated in A549 cells (Korea Cell Line Bank, Seoul, South Korea) using a slight modification of a previously described MTT assay. ${ }^{24-27}$ Cells were seeded in 96-well plates at $10^{5}$ cells/well, incubated
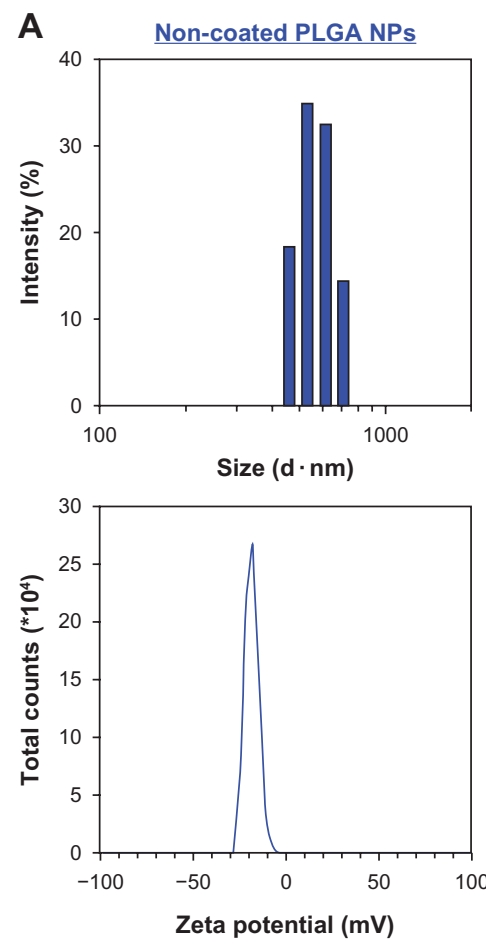

for 12 hours, and then further incubated with suspended chitosan PLGA NPs (final concentration: $0.003-0.3 \mathrm{mg} / \mathrm{mL}$ ) for predetermined times (12 hours). After MTT treatment, cell viabilities were calculated as percentages of non-treated controls. Results are presented as the means \pm standard deviations of at least six independent determinations.

\section{Data analysis}

Results are presented as means \pm standard deviations. Statistical significances were determined using the oneway analysis of variance test, and $P$-values of $<0.05$ were considered significant.

\section{Results}

\section{Preparation and characterization of chitosan PLGA NPs}

PLGA NPs were prepared by emulsification and then surfacecoated with chitosan. Chitosan PLGA NPs had a particle size of $695.7 \pm 62.7 \mathrm{~nm}$, which was greater than that of noncoated PLGA NPs $(593.7 \pm 33.3 \mathrm{~nm})$. The zeta potential of chitosan PLGA NPs was much higher than that of PLGA NPs $(28.5 \pm 0.4 \mathrm{mV}$ versus $-17.9 \pm 0.5 \mathrm{mV})$ (Figure 1). Although the above particle size difference was insignificant $(P>0.07 ; \mathrm{n}=6)$, the mean zeta potential difference was highly significant $(P<0.0001 ; \mathrm{n}=6)$.
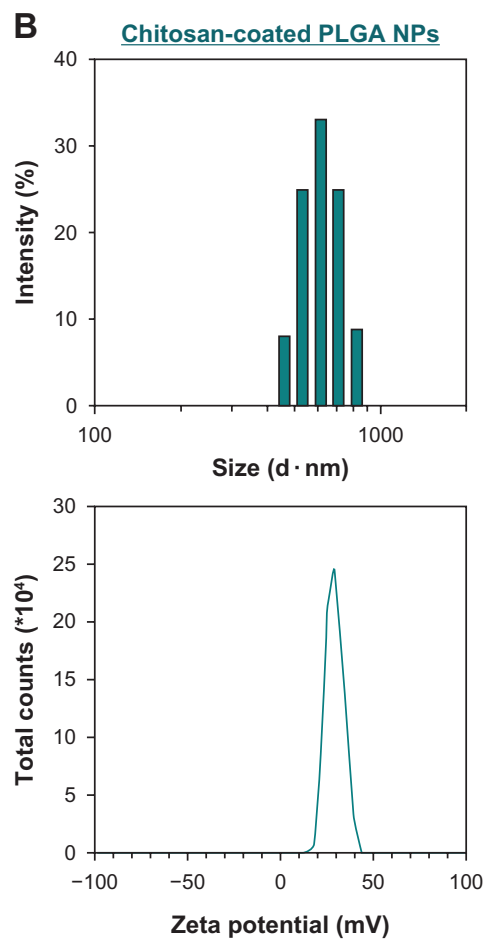

Figure I Histograms of the particle sizes and the zeta potentials of (A) non-coated PLGA NPs and (B) chitosan-coated PLGA NPs. Abbreviation: PLGA NPs, poly(lactic-co-glycolic acid) nanoparticles. 


\section{Surface morphologies of chitosan PLGA NPs}

Chitosan PLGA NPs were found to be spherical and compact in field emission scanning electron microscopy and TEM images (Figure 2), and particle sizes were similar to those measured using the particle size analyzer. The surface morphologies of chitosan PLGA NPs were also similar with non-coated PLGA NPs.

\section{Adsorption of chitosan PLGA NPs by $\mathrm{A} 549$ lung cells}

As shown in Figure 3A, A549 cells treated with non-coated PLGA NPs containing coumarin 6 were much less green, whereas chitosan PLGA NPs containing coumarin 6 were obviously adsorbed by A549 cells, displaying significantly strong green fluorescence. (Figure 3B).

\section{Release of Ex4 and Pal-Ex4 from chitosan PLGA NPs}

The effects of the chitosan-coating on the release profiles of Ex4 and of Pal-Ex4 from chitosan PLGA NPs were assessed. As shown in Figure 4, Ex4 was rapidly released irrespective of the chitosan-coating; in fact, more than $80 \%$ of incorporated Ex4 was lost in 1 day. However, the release of Pal-Ex4 from PLGA NPs was much slower than that of Ex4, and Pal-Ex4 was released more slowly by chitosan-coated PLGA NPs than by non-coated PLGA NPs over the 3-day test period.

\section{Aerosolization of chitosan PLGA NPs}

The aerosolization of chitosan PLGA NPs from the microsprayer was maintained for $\sim 0.40$ seconds after actuation, and the
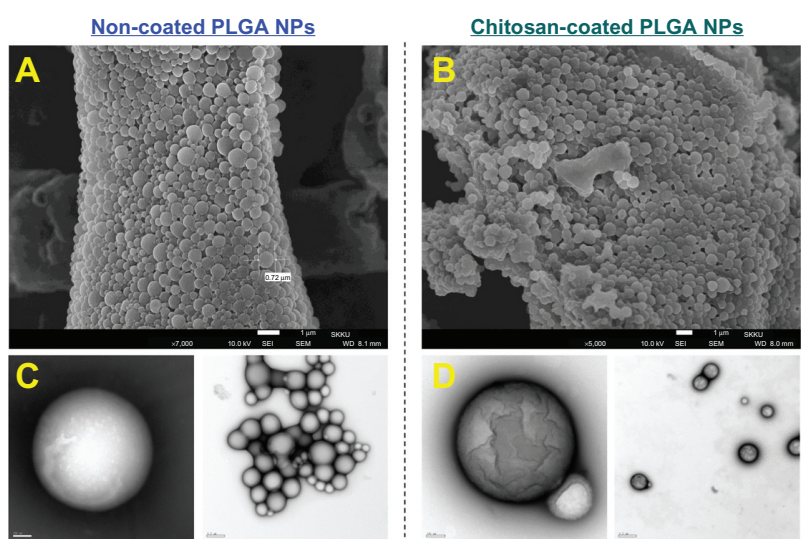

Figure 2 FE-SEM photographs of (A) non-coated PLGA NPs, (B) chitosan-coated PLGA NPs, and TEM images of (C) non-coated PLGA NPs and (D) chitosan-coated PLGA NPs.

Abbreviations: FE-SEM, field emission scanning electron microscopy; PLGA NPs, poly(lactic-co-glycolic acid) nanoparticles; TEM, transmission electron microscopy.
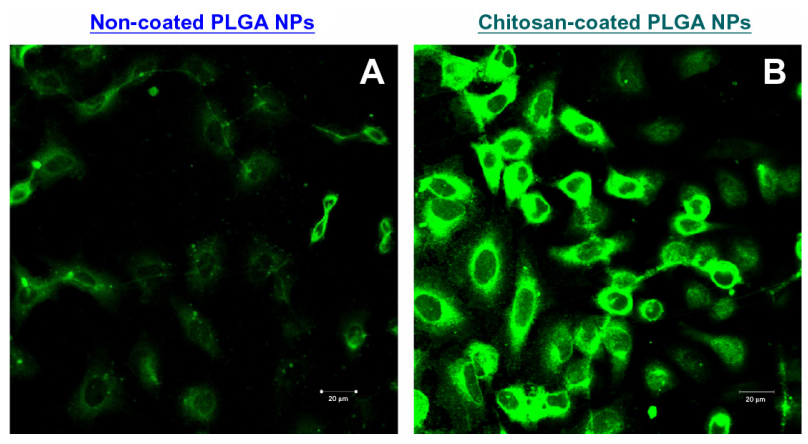

Figure 3 Confocal laser scanning microscopic images of coumarin 6-loaded PLGA NPs: (A) non-coated and (B) chitosan-coated.

Abbreviation: PLGA NPs, poly(lactic-co-glycolic acid) nanoparticles.

aerosolization process was captured at 0.08 second intervals (Figure 5A). Aerosol particles showed good mobility despite high concentrations of chitosan PLGA NPs $(\sim 5 \mathrm{mg} / \mathrm{mL})$ in $10 \mathrm{mM}$ PBS. The mobility of chitosan PLGA NPs did not appear to be affected by the loading of Ex4 or Pal-Ex4.

\section{Lung deposition of PLGA NPs}

Coumarin 6-loaded PLGA NPs with or without a chitosancoating were intubated into the lungs of ICR mice using the microsprayer and an otoscope. Photo images obtained using an in vivo imaging system revealed PLGA NPs in the lungs at 8, 24, and 72 hours after intubation. Chitosan PLGA NPs were found to be rapidly deposited but gradually eliminated over 72 hours, whereas naïve PLGA NPs without a chitosan coating were rapidly cleared and most nanoparticles disappeared at 8 hours (Figure 5B).

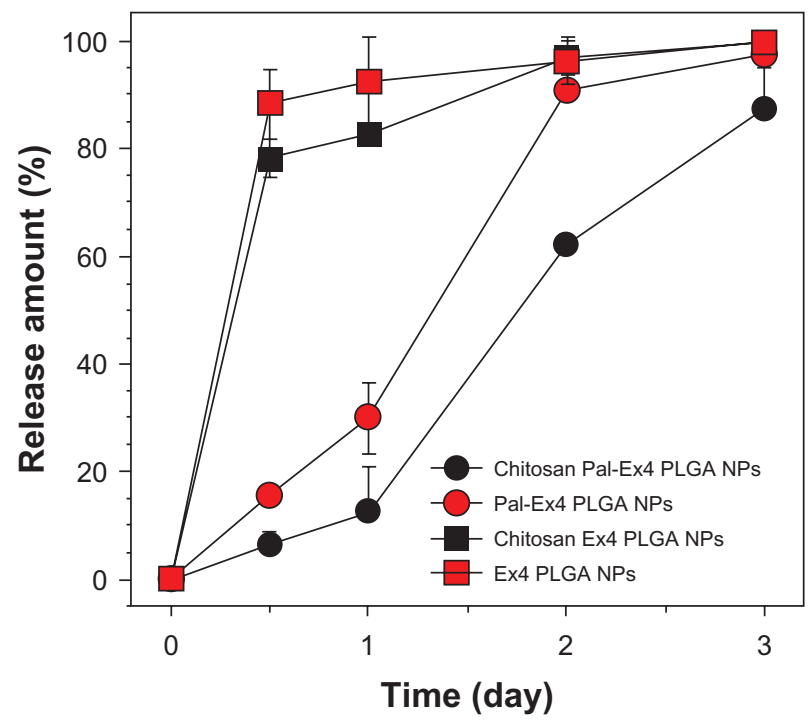

Figure 4 Release profiles of Ex4 and Pal-Ex4 from chitosan-coated or non-coated PLGA NPs.

Abbreviations: Ex4, exendin-4; Pal-Ex4, palmitic acid-modified exendin-4; PLGA NPs, poly(lactic-co-glycolic acid) nanoparticles. 


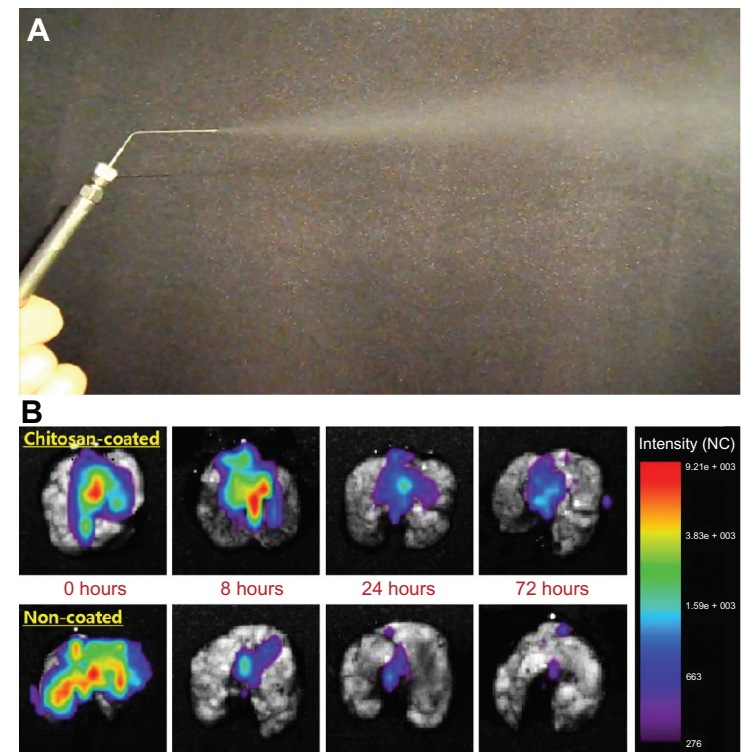

Figure 5 (A) Photograph of aerosolization of chitosan-coated Pal-Ex4 PLGA NPs using a microsprayer aerosolizer; (B) Representative images of lungs excised from ICR mice at various times after the pulmonary administration of chitosan-coated or non-coated PLGA NPs containing coumarin 6.

Abbreviations: ICR, imprinting control region; Pal-Ex4, palmitic acid-modified exendin-4; PLGA NPs, poly(lactic-co-glycolic acid) nanoparticles.

\section{Hypoglycemic efficacy evaluations of chitosan Ex4 or Pal-Ex4 PLGA NPs in non-fasted $\mathrm{db} / \mathrm{db}$ mice after pulmonary administration}

The hypoglycemic efficacies of chitosan Ex4 or Pal-Ex4 PLGA NPs (100 nmol/kg body weight) were examined in non-fasted type 2 diabetic $\mathrm{db} / \mathrm{db}$ mice. As shown in Figure 6, the time to reach minimum glucose level was clearly longer for chitosan Pal-Ex4 PLGA NPs (4 hours versus 24 hours) and the hypoglycemia induced by chitosan Pal-Ex4 PLGA NPs lasted until 48 hours after administration $(206.5 \pm 74.9 \mathrm{mg} / \mathrm{dL})$ as compared with the $>400 \mathrm{mg} / \mathrm{dL}$ observed for chitosan Ex4 PLGA NPs. Furthermore, the hypoglycemia induced by chitosan Pal-Ex4 PLGA NPs was 3.1-fold greater than that induced by chitosan Ex4 PLGA NPs $(17653.6 \pm 2947.7 \mathrm{mg} \cdot \mathrm{h} / \mathrm{dL}$ versus $5669.3 \pm 587.2 \mathrm{mg} \cdot \mathrm{h} / \mathrm{dL}$, respectively) based on the total hypoglycemic degree values described above.

\section{Cytotoxicity of chitosan PLGA NPs in human lung epithelial cells}

The acute cytotoxicity of PLGA NPs was evaluated using A549 cells. As shown in Figure 7, PLGA NPs did not exhibit significant cytotoxicity ( $\sim 95 \%$ cell viability) at 12 hours post-administration at a concentration of $0.03 \mathrm{mg} / \mathrm{mL}$, regardless of the presence of a chitosan-coating. However, both chitosan-coated PLGA NPs and non-coated PLGA NPs appeared to be slightly cytotoxic to A549 cells at concentrations of over $0.03 \mathrm{mg} / \mathrm{mL}$, and thus the cytotoxicity seemed not to be solely based on whether it was coated by chitosan.

\section{Discussion}

PLGA has been intensively used as a matrix for preparing nanoparticles and microspheres in the context of sustained

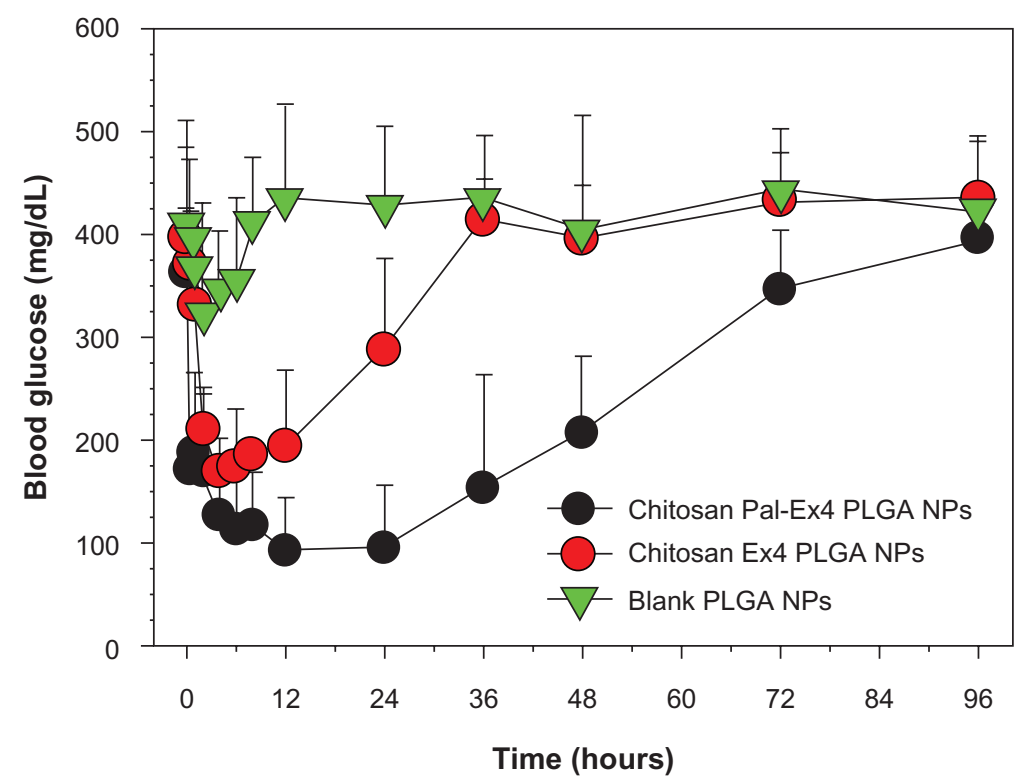

Figure 6 Hypoglycemic efficacies of chitosan-coated PLGA NPs containing Ex4 or Pal-Ex4 in non-fasted db/db mice at a dose of $100 \mathrm{nmol} / \mathrm{kg}$. Notes: Data represent six mice and are presented as means \pm SDs.

Abbreviations: Ex4, exendin-4; Pal-Ex4, palmitic acid-modified exendin-4; PLGA NPs, poly(lactic-co-glycolic acid) nanoparticles; SD, standard deviation. 


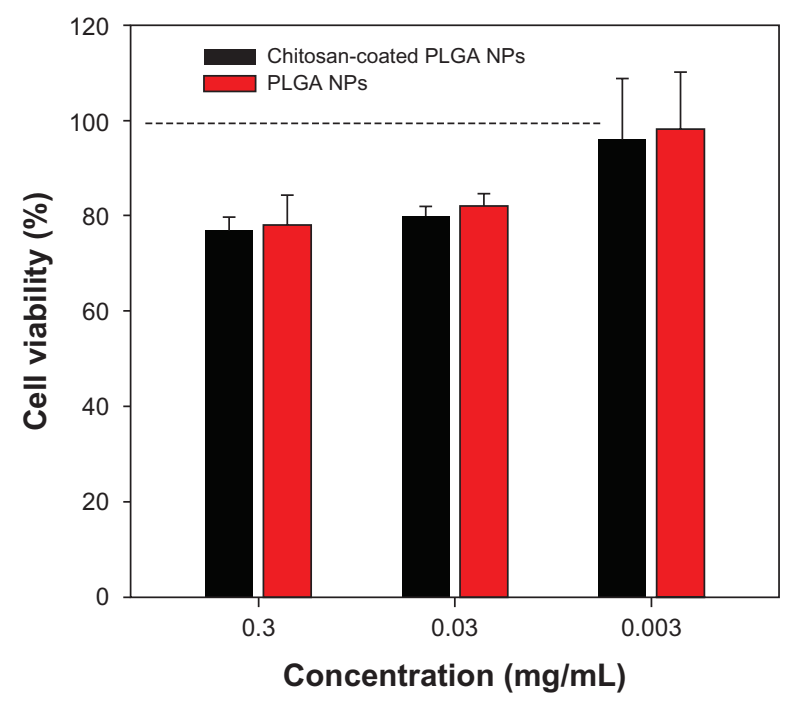

Figure 7 Effects of chitosan-coated PLGA NPs at different concentrations on the viabilities of $A 549$ cells.

Abbreviation: PLGA NPs, poly(lactic-co-glycolic acid) nanoparticles.

protein release. Furthermore, PLGA was approved by the US Food and Drug Administration (FDA) because it is biocompatible and biodegradable, and thus, is believed to be safer than the available alternatives. ${ }^{6,7}$ However, fabricating PLGA nanoparticles $(<200 \mathrm{~nm})$ is not easily achieved because it requires high speed/shear homogenization, and its loading is relatively very low, aside from an initial burst problem. Therefore, its use as intravenously injected nanoparticles is unattractive for targeting tissues. On the other hand, PLGA microspheres $(\sim 1 \mu \mathrm{m})$ are considered more useful for administration by inhalation because they can be dispersed well in an aqueous solution and easily aerosolized using microsprayer-like nebulizers or metered dose inhalers. ${ }^{13,28} \mathrm{In}$ this regard, we chose PLGA nanoparticles that can be loaded with proteins and then released in a sustained manner as a delivery tool for Pal-Ex4 in order to devise a long-acting inhalatory anti-diabetic system.

Despite the merits of nanoparticles with respect to the inhalatory route, PLGA nanoparticles cannot remain in situ for long due to electrical repulsion between the negative charges of PLGA and lung cell membranes. Thus, a chitosancoating was considered to introduce positive charges on PLGA in many studies. However, chitosan has a $\mathrm{pK}_{\mathrm{a}}$ value of 5.5-6.5, and thus, it is likely to aggregate and lose its positive charges at physiological $\mathrm{pH}$ values. ${ }^{13,14,29}$ On the other hand, glycol chitosan, an ethylene glycol conjugated form, is known to have greater water solubility and to retain its positive charges. ${ }^{12}$ Consequently, glycol chitosan is more mucoadhesive due to increased electrostatic attraction, hydrogen bonding, and hydrophobicity. ${ }^{30}$ In the present study, chitosan-coated PLGA NPs were found to be better adsorbed by A549 cells (Figure 3).

In the present study, chitosan-coated PLGA NPs were fabricated in a conventional manner, and increasing homogenization speed (to a few tens of thousands of rpm) was found to decrease particle size. However, excessive homogenization speeds $(>20,000 \mathrm{rpm})$ are apt to result in rapid temperatures, and thus, could harm the molecular integrity of the incorporated Ex4. Furthermore, small nanoparticles of few hundred nanometers were not considered appropriate for inhalation, and thus, homogenization speed was set at $<10,000 \mathrm{rpm}$. Under the conditions used, the particle sizes of the resulting nanoparticles were around $600-700 \mathrm{~nm}$, and their shapes and homogeneity appeared excellent. Nevertheless, the incorporation efficiencies of Ex4 and of Pal-Ex4 into chitosan-coated PLGA NPs were not high (33.4 and 23.1\%, respectively). As reported previously, Pal-Ex4 tends to associate and form micelles at concentrations $>40 \mu \mathrm{g} / \mathrm{mL},{ }^{6,7}$ and we believe that this explains its lower incorporation.

However, although the incorporation of Pal-Ex4 into PLGA was relatively low, Pal-Ex4 release was found to be delayed by as much as $>1.5$ days versus Ex4, especially for chitosan-coated PLGA NPs, which was attributed to the hydrophobic interaction between the palmityl group of Pal-Ex4 and the lactic/glycolic acid moiety of PLGA. ${ }^{7}$ Consequently, the initial burst release shown by Ex4 PLGA NPs was not observed for Pal-Ex4 PLGA NPs. Separately, the Pal-Ex4 release from chitosan PLGA NPs was slower than that from PLGA NPs possibly because Pal-Ex4 release was inhibited by the chitosan-coating due to the ionic interaction between Ex4 and chitosan. In addition, chitosan-coated Pal-Ex4 PLGA NPs displayed good aerosolization for inhalatory properties when administered using a microsprayer (Figure 5).

The longevity of chitosan-coated PLGA NPs in lung was investigated by infrared fluorescence imaging in mice. Generally, Cy5.5 NHS has been intensively used for imaging, but in the present study was ruled out because of its poor loading efficiency into PLGA NPs when organic solvents, such as methylene chloride, were used for the preparation of the nanoparticles. Instead, a fluorescent dye, coumarin 6, was used to visualize nanoparticles deposition in lungs because considerable amounts could be incorporated into PLGA NPs. Actually, coumarin 6-loaded PLGA NPs were well visualized in lungs, and our imaging results showed that chitosan PLGA NPs remained in place much longer than non-coated PLGA NPs.

The use of palmitic acid-modified Ex4 also provides advantages to the chitosan Pal-Ex4 PLGA NPs. In general, 


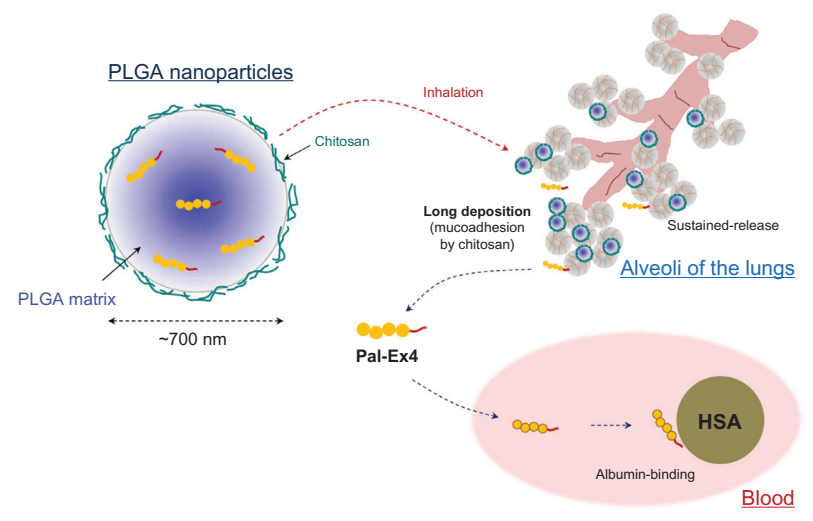

Figure 8 An illustration of the rationale underlying the design of the chitosan-coated PLGA NPs containing Pal-Ex4.

Abbreviations: HSA, human serum albumin; Pal-Ex4, palmitic acid-modified exendin-4; PLGA NPs, poly(lactic-co-glycolic acid) nanoparticles.

fatty acid-modified peptides bind tightly to serum albumin molecules due to a strong attraction between their fatty acid components and albumin at several docking sites..$^{31,32}$ Fattyacid modified peptides have longer circulatory life times than their unmodified counterparts. This conjugation technology has been used to extend the therapeutic efficacies of many peptides, such as Levemir ${ }^{\circledR}$ and Victoza ${ }^{\circledR}$ (Novo Nordisk, Bagsværd, Denmark), which are myristic acid- and palmitic acid-modified insulin and glucagon-like peptide-1 analogs, respectively, that have been approved as extended antidiabetic treatments by the FDA. ${ }^{7,31}$ In this regard, Pal-Ex4 was incorporated into chitosan-coated PLGA NPs to extend hypoglycemia after release from nanoparticles. In the present study, Pal-Ex4 PLGA NPs showed a significantly more protracted hypoglycemic effect than Ex4 PLGA NPs ( 2 days versus $\sim 0.5$ days).

Although PLGA and glycol chitosan are considered to be relatively safe, the long-term deposition of glycol chitosan PLGA could induce inflammation or acute toxicity. In fact, some nanoparticles made of or coated with chitosan have exhibited acute moderate cytotoxicity in vitro at concentrations of $\sim 0.01 \mathrm{mg} / \mathrm{mL}^{26,27}$ and mild proinflammatory responses in rat and mouse lungs. ${ }^{25,33}$ Likewise, our chitosancoated PLGA NPs were found to be slightly cytotoxic to A549 lung cells in vitro. However, in vitro cell toxicity can also cause overestimations because conditions remain static for long times. ${ }^{24}$ Therefore, careful systemic toxicity testing of chitosan-coated PLGA NPs administered by inhalation is required in the near future.

\section{Conclusion}

Inhalable palmitic acid-modified Ex4 loaded chitosan-coated PLGA nanoparticles were examined with the objective of developing an anti-diabetic inhalatory treatment. This chitosan Pal-Ex4 PLGA NPs system displayed good aerosolization characteristics and protracted hypoglycemic effects in a diabetic rodent model after pulmonary administration. As illustrated in Figure 8, we believe this improvement was caused by the enhancements of (1) the mucoadhesion effect of the chitosan surface coat, (2) the sustained-release effects of PLGA and palmitic acid, and (3) the circulatory longevity of released Pal-Ex4 due to albumin binding. We believe that this inhalation system has considerable pharmaceutical potential in treatment for type 2 diabetes.

\section{Acknowledgments}

This work was supported by the Korean National Research Foundation (NRF) supported by the Korea government (MEST) (\#20120005385), and by the Korean Health Technology R\&D Project, Ministry for Health, Welfare and Family Affairs (\#A092018).

\section{Disclosure}

The authors report no conflicts of interest in this work.

\section{References}

1. Patton JS, Byron PR. Inhaling medicines: delivering drugs to the body through the lungs. Nat Rev Drug Discov. 2007;6(1):67-74.

2. Patton JS, Fishburn CS, Weers JG. The lungs as a portal of entry for systemic drug delivery. Proc Am Thorac Soc. 2004;1(4):338-344.

3. Patton JS. Mechanisms of macromolecule absorption by the lungs. $A d v$ Drug Deliv Rev. 1996;19(1):3-36.

4. Lee J, Lee C, Kim I, et al. Preparation and evaluation of palmitic acid-conjugated exendin- 4 with delayed absorption and prolonged circulation for longer hypoglycemia. Int J Pharm. 2012;424(1-2):50-57.

5. Lee J, Lee C, Kim TH, et al. Pulmonary administered palmitic-acid modified exendin- 4 peptide prolongs hypoglycemia in type 2 diabetic $\mathrm{db} / \mathrm{db}$ mice. Regul Pept. 2012;177(1-3):68-72.

6. Kim H, Lee J, Kim TH, et al. Albumin-coated porous hollow poly(lacticco-glycolic acid) microparticles bound with palmityl-acylated exendin-4 as a long-acting inhalation delivery system for the treatment of diabetes. Pharm Res. 2011;28(8):2008-2019.

7. Kim H, Park H, Lee J, et al. Highly porous large poly(lactic-co-glycolic acid) microspheres adsorbed with palmityl-acylated exendin-4 as a long-acting inhalation system for treating diabetes. Biomaterials. 2011;32(6):1685-1693.

8. Youn YS, Lee KC, Bae YH, Na K, Lee ES. Advanced pulmonary delivery of peptides or proteins using polymeric particles. In: Jorgensen L, Nielsen HM, editors. Delivery Technologies for Biopharmaceuticals: Peptides, Proteins, Nucleic Acids, and Vaccines. Hoboken: Wiley; 2009:228-244.

9. Sung JC, Pulliam BL, Edwards DA. Nanoparticles for drug delivery to the lungs. Trends Biotechnol. 2007;25(12):563-570.

10. Yang R, Shim WS, Cui FD, et al. Enhanced electrostatic interaction between chitosan-modified PLGA nanoparticle and tumor. Int J Pharm. 2009;371(1-2):142-147.

11. Lauweryns JM, Baert JH. Alveolar clearance and the role of the pulmonary lymphatics. Am Rev Respir Dis. 1977;115(4):625-683.

12. Makhlof A, Werle M, Tozuka Y, Takeuchi H. Nanoparticles of glycol chitosan and its thiolated derivative significantly improved the pulmonary delivery of calcitonin. Int J Pharm. 2010;397(1-2):92-95. 
13. Yamamoto H, Kuno Y, Sugimoto S, Takeuchi H, Kawashima Y. Surface-modified PLGA nanosphere with chitosan improved pulmonary delivery of calcitonin by mucoadhesion and opening of the intercellular tight junctions. J Control Release. 2005;102(2):373-381.

14. Zhang Q, Shen Z, Nagai T. Prolonged hypoglycemic effect of insulin-loaded polybutylcyanoacrylate nanoparticles after pulmonary administration to normal rats. Int J Pharm. 2001;218(1-2):75-80.

15. Censi R, Di Martino P, Vermonden T, Hennink WE. Hydrogels for protein delivery in tissue engineering. J Control Release. 2012;161(2): 680-692.

16. Bhattarai N, Gunn J, Zhang M. Chitosan-based hydrogels for controlled, localized drug delivery. Adv Drug Deliv Rev. 2010;62(1):83-99.

17. Park JH, Saravanakumar G, Kim K, Kwon IC. Targeted delivery of low molecular drugs using chitosan and its derivatives. Adv Drug Deliv Rev. 2010;62(1):28-41.

18. Chae SY, Choi YG, Son S, Jung SY, Lee DS, Lee DS. The fatty acid conjugated exendin-4 analogs for type 2 antidiabetic therapeutics. J Control Release. 2010;144(1):10-16.

19. Chae SY, Jin CH, Shin JH, et al. Biochemical, pharmaceutical and therapeutic properties of long-acting lithocholic acid derivatized exendin-4 analogs. J Control Release. 2010;142(2):206-213.

20. Zhang X, Sun M, Zheng A, Cao D, Bi Y, Sun J. Preparation and characterization of insulin-loaded bioadhesive PLGA nanoparticles for oral administration. Eur J Pharm Sci. 2012:45(5):632-638.

21. Takeuchi H, Yamamoto H, Kawashima Y. Mucoadhesive nanoparticulate systems for peptide drug delivery. Adv Drug Deliv Rev. 2001;47(1): 39-54.

22. Tahara K, Sakai T, Yamamoto H, Takeuchi H, Hirashima N, Kawashima Y. Improved cellular uptake of chitosan-modified PLGA nanospheres by A549 cells. Int J Pharm. 2009;382(1-2):198-204.

23. Tahara K, Yamamoto H, Kawashima Y. Cellular uptake mechanisms and intracellular distributions of polysorbate 80 -modified poly (D,L-lactideco-glycolide) nanospheres for gene delivery. Eur J Pharm Biopharm 2010;75(2):218-224.
24. Lee J, Lee C, Kim TH, et al. Self-assembled glycol chitosan nanogels containing palmityl-acylated exendin-4 peptide as a long-acting anti-diabetic inhalation system. J Control Release. 2012;161(3): 728-734.

25. Choi M, Cho M, Han BS, et al. Chitosan nanoparticles show rapid extrapulmonary tissue distribution and excretion with mild pulmonary inflammation to mice. Toxicol Lett. 2010;199(2):144-152.

26. Nafee N, Schneider M, Schaefer UF, Lehr CM. Relevance of the colloidal stability of chitosan/PLGA nanoparticles on their cytotoxicity profile. Int J Pharm. 2009;381(2):130-139.

27. Grenha A, Grainger CI, Dailey LA, et al. Chitosan nanoparticles are compatible with respiratory epithelial cells in vitro. Eur J Pharm Sci. 2007;31(2):73-84.

28. Kawashima Y, Yamamoto H, Takeuchi H, Fujioka S, Hino T. Pulmonary delivery of insulin with nebulized DL-lactide/glycolide copolymer (PLGA) nanospheres to prolong hypoglycemic effect. J Control Release. 1999;62(1-2):279-287.

29. Chakravarthi SS, Robinson DH. Enhanced cellular association of paclitaxel delivered in chitosan-PLGA particles. Int J Pharm. 2011;409(1-2): 111-120.

30. Sogias IA, Williams AC, Khutoryanskiy VV. Why is chitosan mucoadhesive. Biomacromolecules. 2008;9(7):1837-1842.

31. Kratz F. Albumin as a drug carrier: design of prodrugs, drug conjugates and nanoparticles. J Control Release. 2008;132(3):171-183.

32. Chuang VT, Kragh-Hansen U, Otagiri M, Pharmaceutical strategies utilizing recombinant human serum albumin. Pharm Res. 2002;19(5): 569-577.

33. Huang YC, Vieira A, Huang KL, Yeh MK, Chiang CH. Pulmonary inflammation caused by chitosan microparticles. J Biomed Mater Res A 2005;75(2):283-287.
International Journal of Nanomedicine

\section{Publish your work in this journal}

The International Journal of Nanomedicine is an international, peerreviewed journal focusing on the application of nanotechnology in diagnostics, therapeutics, and drug delivery systems throughou the biomedical field. This journal is indexed on PubMed Central, MedLine, CAS, SciSearch $₫$, Current Contents $₫ /$ Clinical Medicine,

\section{Dovepress}

Journal Citation Reports/Science Edition, EMBase, Scopus and the Elsevier Bibliographic databases. The manuscript management system is completely online and includes a very quick and fair peer-review system, which is all easy to use. Visit http://www.dovepress.com/ testimonials.php to read real quotes from published authors 